\title{
Prognostic Factors for Venous Thromboembolism in Patients with Solid Tumours on Systemic Therapy: A Systematic Review
}

\author{
Sandra Lee ${ }^{1}$ Anika Shenoy $^{2}$ Daniel Shi ${ }^{3}$ Mootaz Husien ${ }^{4}$ Pablo E. Serrano ${ }^{1} \quad$ Sameer Parpia ${ }^{5,6}$
}

${ }^{1}$ Department of Surgery, McMaster University, Hamilton, Ontario,

Address for correspondence Sameer Parpia, PhD, Juravinski Hospital Canada \& Cancer Centre, 711 Concession St - G wing, Room 125, Hamilton,

2 Hamilton Health Sciences, Hamilton, Ontario, Canada

${ }^{3}$ Department of Medicine, Queen's University, Kingston, Ontario, ON, L8V 1C3, Canada (e-mail: parpia@mcmaster.ca).

Canada

${ }^{4}$ University of Waterloo, Waterloo, Ontario, Canada

${ }^{5}$ Department of Oncology, McMaster University, Hamilton, Ontario, Canada

${ }^{6}$ Department of Health Research Methods, Evidence, and Impact, McMaster University, Hamilton, Ontario, Canada

TH Open 2021;5:e461-e469.

\begin{abstract}
Keywords

- venous thromboembolism

- chemotherapy

- neoplasms

- prognosis

Background Patients undergoing systemic cancer therapy are susceptible to developing venous thromboembolism (VTE). The most pertinent prognostic factors for VTE remain unclear. This systematic review aims to summarize prognostic factors associated with VTE in this population.

Methods MEDLINE, Embase, and CENTRAL databases were searched for observational or randomized studies that used multivariable analysis adjusted for tumor type and/or metastatic disease to model the risk of VTE. Adjusted effect estimates for each prognostic factor were collected for all of the included studies. Risk of bias was assessed using the Quality in Prognostic Factor Studies (QUIPS) tool.

Results From 5,988 search results, 15 eligible studies and 42 prognostic factors were identified. A total of 8,554 patients of whom 456 (5.33\%) developed VTE were included. Fourteen studies had a high risk of bias and one study had a moderate risk. The most commonly reported prognostic factors include age, gender, tumor site, metastasis, performance status, and systemic therapy type. Poor performance status and the use of platinum-based chemotherapy compounds were associated with an increased risk of VTE across the majority of studies. The evidence to suggest that the other prognostic factors identified were associated with VTE development was inconclusive. Several individual studies identified novel biomarkers for VTE. Heterogeneity in statistical methods and prognostic factor definitions across studies precluded meta-analysis. Conclusion Overall, many prognostic factors were identified; however, the evidence for association with development of VTE for most of the factors is inconclusive. Findings were limited by high heterogeneity and risk of bias in the included studies.
\end{abstract}

received

April 27, 2021

accepted after revision

September 7, 2021
DOI https://doi.org/

10.1055/a-1642-4572.

ISSN 2512-9465. (c) 2021. The Author(s).

This is an open access article published by Thieme under the terms of the Creative Commons Attribution License, permitting unrestricted use, distribution, and reproduction so long as the original work is properly cited. (https://creativecommons.org/licenses/by/4.0/)

Georg Thieme Verlag KG, Rüdigerstraße 14, 70469 Stuttgart, Germany 


\section{Introduction}

Venous thromboembolism (VTE) is a common complication among patients with cancer, particularly in those receiving systemic chemotherapy or other antineoplastic treatments. ${ }^{1,2}$ The incidence of VTE ranges from $2 \%$ to $8 \%$ in this population and has been identified as a leading cause of death in these patients. ${ }^{3-6}$ Certain factors have been identified that further exacerbate the risk of VTE. For instance, certain solid tumors are associated with the highest risk of VTE, namely, pancreatic, stomach, and lung cancers. ${ }^{1,4,6,7}$ Additionally, the presence of metastatic disease has been identified as a significant prognostic factor, increasing the risk of VTE by 4 -fold to 13 -fold compared with earlier stages. ${ }^{1,4}$

The identification of such prognostic factors allows patients to be stratified into risk groups and aid in decisions to administer thromboprophylaxis prior to cancer treatment. As such, a widely used and validated scoring model, the Khorana score, predicts VTE in patients receiving chemotherapy on the basis of five clinical and laboratory parameters. ${ }^{7}$ These include tumor site, body mass index, hemoglobin levels, platelet count, and leukocyte count. ${ }^{7}$ Nonetheless, identifying patients who are at a high risk for VTE remains a challenge. A recent meta-analysis looked at 6-month VTE incidence by Khorana risk category in 27,849 cancer patients. $^{8}$ This study found that most VTE events occurred in the low- and intermediate-risk groups (11.6\%) rather than the high-risk group (11.0\%). Of the patients who were diagnosed with VTE, $23.4 \%$ were classified as high-risk according to the Khorana score. ${ }^{8}$ Newer studies have since identified additional prognostic factors, including predictive biomarkers, for VTE. For instance, Ay et al. observed that adding D-dimer and soluble P-selectin as variables to the Khorana score more accurately predicted VTE. ${ }^{9}$ Additionally, certain types of chemotherapy regimens were found to significantly increase the risk of VTE. ${ }^{10}$

Although many studies have investigated various clinical and pathological factors that predict VTE, these results have yet to be systematically examined. Therefore, this systematic review was conducted to identify and summarize the most pertinent prognostic factors associated with the development of VTE in patients with solid tumors undergoing systemic therapy.

\section{Methods}

This systematic review was conducted and reported according to the Preferred Reporting for Systematic Reviews and Meta-Analyses (PRISMA) guidelines. ${ }^{11}$ The protocol of this study was registered prior to study commencement in the Prospective Register of Systematic Reviews (CRD42020165501).

\section{Search Strategy}

A comprehensive search of MEDLINE, Embase, and the Cochrane Central Register of Controlled Trials (CENTRAL) databases was conducted, obtaining records from database conception to August 2019 inclusive. Highly sensitive search strategies unique to each database were developed (-Supplementary Table S1-3). Subject headings and key words were used to form the strategy, which included terms such as venous thromboembolism, cancer, systemic therapy, and their synonyms. These phrases were combined with the methodology term 'prognosis'. References of included studies were hand-searched to identify any additional relevant studies.

\section{Eligibility}

Studies were eligible for inclusion if they reported a multivariable analysis to model the risk of VTE in patients with solid tumors undergoing chemotherapy or other types of systemic therapy. VTE was defined as symptomatic and incidental pulmonary embolism and deep vein thrombosis. Only patients with solid tumors, including lymphomas, were included in this review. Primary brain tumors and leukemias were excluded due to differences in treatment course and staging compared with other solid tumors. Additionally, patients who received thromboprophylaxis or underwent surgery during the treatment or follow-up period were excluded. Studies of any study design available in English were included.

\section{Data Abstraction}

Titles and abstracts were screened by two independent reviewers using pre-defined inclusion and exclusion criteria. Full-text review was also conducted by two independent reviewers, with the most important reason for exclusion documented during this stage. Conflicts at each stage were resolved through discussion and consensus. A third reviewer was consulted if consensus could not be reached. Agreement between reviewers was calculated using Cohen's kappa (k) for each step of the screening process.

Data was extracted by two independent reviewers using a standardized, pilot-tested form designed a priori. Study characteristics, patient demographics, tumor characteristics, treatment types, VTE incidence, and all prognostic factors reported by each study were recorded. For studies that performed multivariable analysis on a subset of patients, only the subset was considered for this review. Adjusted effect estimates for each prognostic factor were drawn, which were determined using odds ratios (ORs), hazard ratios (HRs), and sub-distributed hazard ratios (SHRs). For studies that did not report full multivariable analysis results, corresponding authors were contacted for this information. To avoid double-counting patients that were included in more than one study, corresponding authors of studies conducted by the same research group were contacted for clarification around overlapping cohorts.

\section{Selection of Prognostic Factors}

The purpose of this review was to identify the most pertinent prognostic factors for VTE, thus, only studies with adjusted effect estimates as modeled in multivariable analyses were included. To further standardize the effect estimates collected for each prognostic factor, only studies that adjusted for at 
least one of the two factors (i.e., tumor site and/or metastasis) were included. These factors were selected for their strong association with VTE development according to previous literature. ${ }^{1,4,12}$ Studies with effect estimates that did not adjust for metastasis and/or tumor type were excluded from this review, as well as studies that did not report a measure of association for prognostic variables.

\section{Statistical Analysis}

Baseline patient characteristics were summarized using descriptive statistics based on the available data in the included studies. If multiple studies reported on overlapping patient cohorts, the study with the largest sample size from each research group was prioritized when calculating baseline characteristics.

Meta-analysis of prognostic factors using the DerSimonian and Laird random effects approach was considered if a prognostic factor was reported in four or more studies using the same effect measure.

\section{Risk of Bias Assessment}

The quality of each study was assessed using the Quality in Prognostic Factor Studies (QUIPS) tool. ${ }^{13}$ The QUIPS tool assigns the risk of bias as low, moderate, or high in six domains: study participation, study attrition, prognostic factor measurement, outcome measurement, study confounding, and statistical analysis and reporting. The domain with the highest risk of bias was used to determine the overall study quality.

\section{Results}

\section{Study Characteristics}

The search strategy identified 5,988 studies of which 111 underwent full-text review. Fifteen studies were eligible for final inclusion (-Fig. 1). A descriptive summary of the included studies can be found in -Table 1. There was substantial agreement for both title and abstract screening $(\mathrm{k}=0.64)$ and full-text review $(\mathrm{k}=0.79)$.

The included studies were published between 2005 and 2019, with six of the 15 studies published in the past five years. Five studies were conducted in Italy by the same research group, ${ }^{14-18}$ two studies in the United States by another research group, ${ }^{7,19}$ and two studies with centres in the Netherlands, Italy, Mexico, and France were conducted by another research group. ${ }^{20,21}$ For the remaining studies, one was conducted in the Netherlands, ${ }^{22}$ one in Jordan, ${ }^{23}$ one in the United States, ${ }^{24}$ two in Italy, ${ }^{25,26}$ and one study with centres across France, Lebanon, Jordan, Saudi Arabia, Kuwait, and Syria. ${ }^{27}$ Nine studies were prospective cohorts, $7,15,18-21,24,25,27$ and 6 studies were retrospective cohorts. $^{14,16,17,22,23,26}$

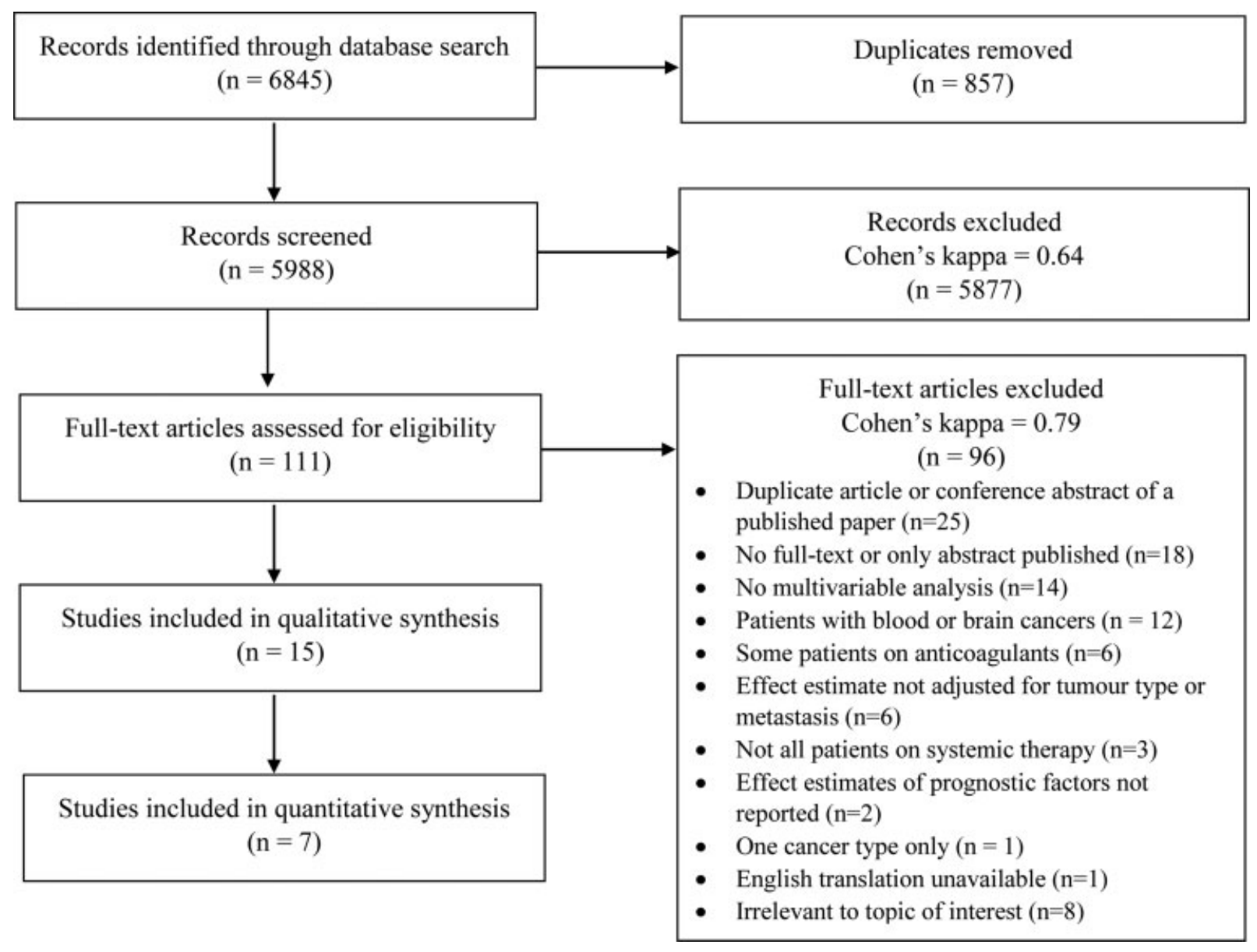

Fig. 1 Flow diagram of study selection. 
e464 Predictors of Venous Thromboembolism in Cancer Therapy Patients Lee et al.

Table 1 Characteristics of included studies

\begin{tabular}{|c|c|c|c|c|}
\hline Author and year & Study design & Country & Sample size & Median follow-up (months) \\
\hline Abdel-Razeq $2018^{23}$ & Retrospective & Jordan & 1677 & $\begin{array}{l}>4 \text { weeks, median not } \\
\text { specified }\end{array}$ \\
\hline Arpaia $2009^{25}$ & Prospective & Italy & 124 & 6 , median not specified \\
\hline Di Nisio $2019^{22}$ & Retrospective & Netherlands & 776 & 10.8 (IQR 5.2-12) \\
\hline Ferroni (GFR) $2014^{14 *}$ & Retrospective & Italy & 322 & 9.3 \\
\hline Ferroni (MPV) $2014^{15 *}$ & Prospective & Italy & 589 & 8.5 \\
\hline Ferroni $2015^{17 *}$ & Retrospective & Italy & 810 (380 in analysis) & 9.2 \\
\hline Ferroni $2016^{16 *}$ & Retrospective & Italy & 297 & 14 \\
\hline Gerotziafas $2017^{27}$ & Prospective & $\begin{array}{l}\text { France, Lebanon, Jordan, } \\
\text { Saudi Arabia, } \\
\text { Kuwait, Syria }\end{array}$ & 1023 & 6, median not specified \\
\hline Khorana $2005^{19_{* *}}$ & Prospective & United States & 3003 & 2.4 \\
\hline Khorana $2008^{7 * *}$ & Prospective & United States & 4066 (2701 in analysis) & 2.4 (range $0.2-12.0$ ) \\
\hline Roselli $2013^{18 *}$ & Prospective & Italy & 505 & 11.2 \\
\hline Tafur $2015^{24}$ & Prospective & United States & 241 & Mean 10.4 (SD 3.2) \\
\hline van Es $2017^{20 * * *}$ & Prospective & $\begin{array}{l}\text { Netherlands, Italy, } \\
\text { Mexico, France }\end{array}$ & 876 & 6 , median not specified \\
\hline van Es $2018^{21_{* * *}}$ & Prospective & $\begin{array}{l}\text { Netherlands, Italy, } \\
\text { Mexico, France }\end{array}$ & 648 & 5.9 (IQR 3.2-5.9) \\
\hline Vergati $2013^{26}$ & Retrospective & Italy & 486 & 12 \\
\hline
\end{tabular}

Abbreviations: IQR, interquartile range; SD, standard deviation.

*Study conducted by same research group.

**Study conducted by same research group.

${ }^{* * *}$ Study conducted by same research group.

\section{Assessment of Quality}

All studies were noted to have some degree of methodological flaws (-Supplementary Table S4). In the majority of studies, the process of identifying and selecting participant was unclear, resulting in a high risk of bias in study participation. There was moderate to high risk of bias for study attrition as the loss to follow-up rates and reasons were not explicitly described in most studies. Consideration of all prognostic factors, including variations in treatment, was incomplete in most studies, resulting in a moderate to high risk of bias for study confounding. Most studies did not provide sufficient detail describing the analysis models, such as how variables were defined in the analysis and the selection process for the variables that were included in the model, which led to a moderate to high risk of bias for statistical analysis and reporting.

\section{Patient Characteristics}

Baseline characteristics of each included study are available in - Table 2. Considering only the study with the largest sample size when multiple studies reported on overlapping patient cohorts, this review includes a total of 8,554 patients of which 456 (5.33\%) developed VTE. The median follow-up time in each study ranged from 2 to 14 months. The mean age ranged from 50 to 65 years old across studies. Of the included patients, the most common types of cancers that were reported were breast (27.9\%), lung (20.5\%), and colorectal
(14.7\%). Overall, 3,967 (46.4\%) of patients had metastatic disease. Of the studies that reported baseline Khorana score risk category, $1,313(30.1 \%)$ patients were at low risk (0 points), 2,538 (58.2\%) at intermediate risk (1-2 points) and $510(11.7 \%)$ at high risk ( $3+$ points). Of the studies that reported baseline Eastern Cooperative Oncology Group (ECOG) performance status, 4,855 (82.7\%) patients had a status of $0-1$ points and $416(7.1 \%)$ patients had a status of $2-4$ points.

\section{Prognostic Factors for VTE}

From the included studies, a total of 42 unique prognostic factors for VTE were identified. - Table 3 lists the classification and frequency of each prognostic factor explored in the included studies. The multivariable analysis results of each prognostic factor identified in this review are available in -Supplementary Table S5. Due to the heterogeneity in patient population, outcomes reported, definition of prognostic factors, and statistical methods used across studies, we opted against meta-analyzing the data and provide a narrative synthesis.

\section{Patient Factors}

The most commonly reported patient-related prognostic factors were age, gender, and ECOG performance status. Age was analyzed in eight studies (or four patient cohorts). 


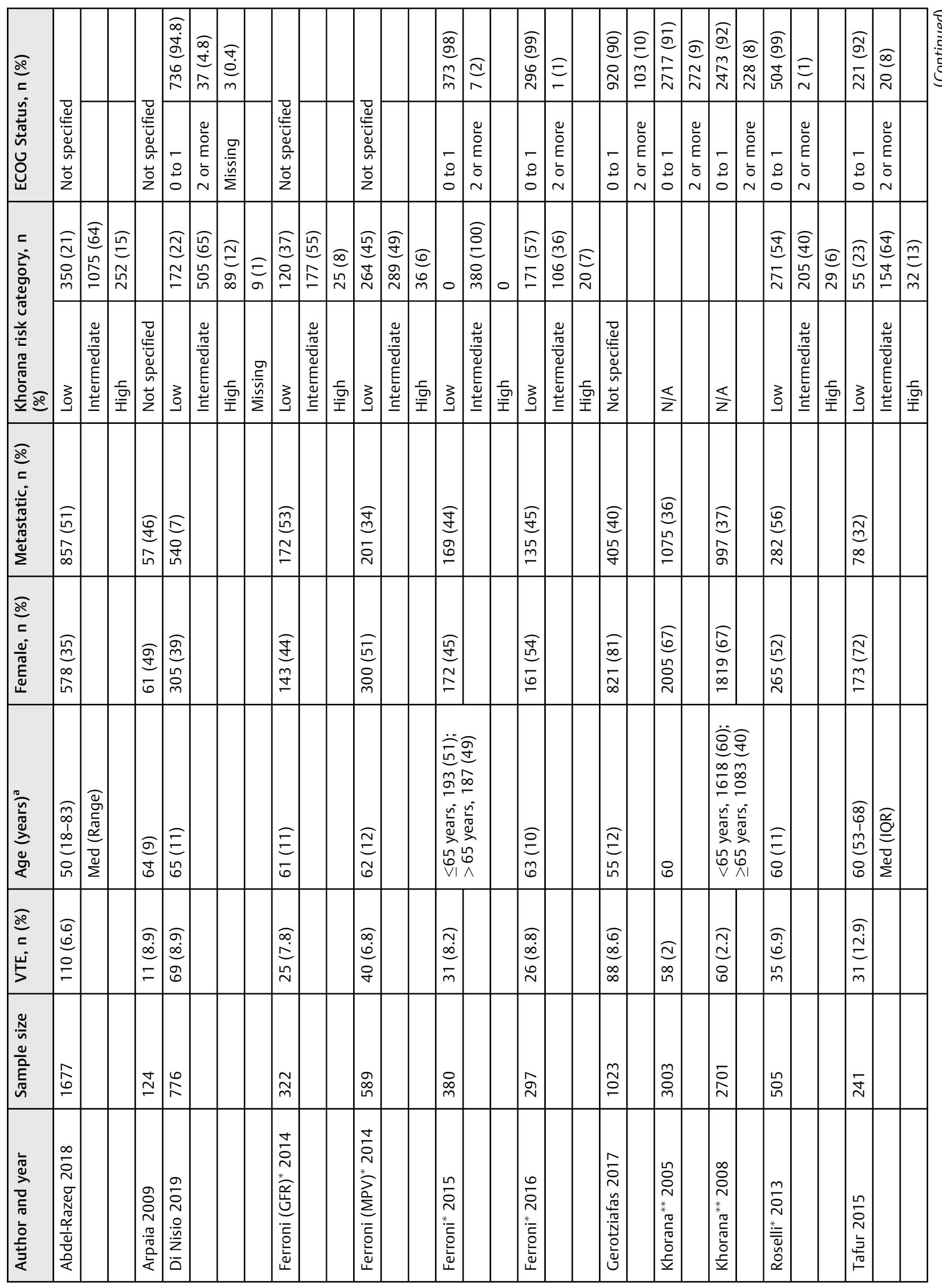




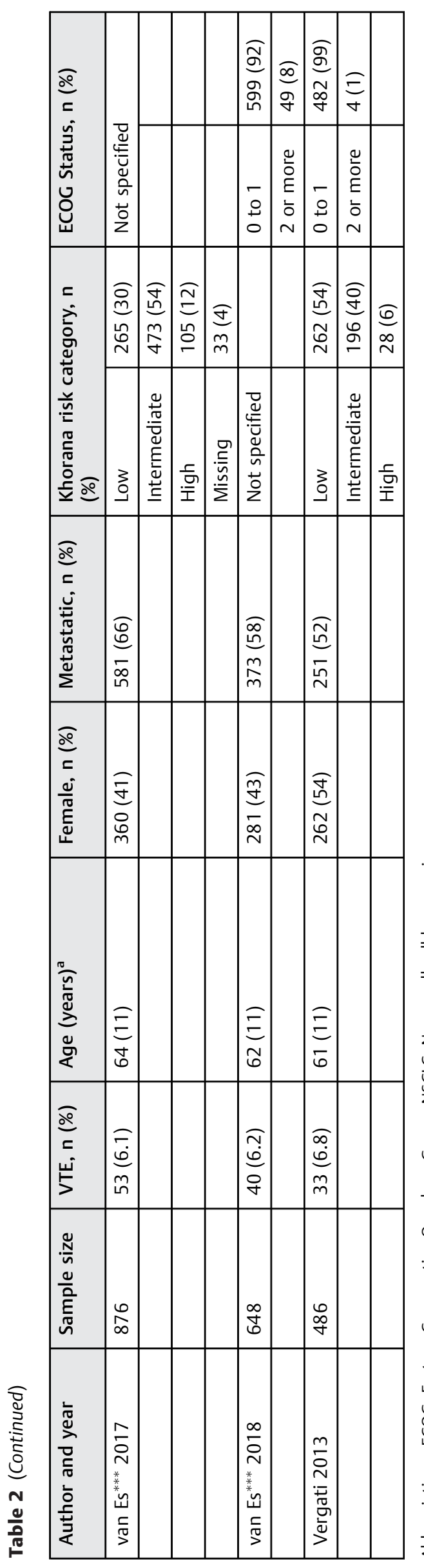

One retrospective cohort study found older age to be a predictor of VTE. ${ }^{26}$ Sex was explored in eight studies (or four patient cohorts). Female was associated with an increased risk of VTE in one retrospective study, ${ }^{23}$ however, the remaining studies found no evidence of association. Eight studies (or four patient cohorts) examined ECOG performance status. Five of these studies (or two patient cohorts) found poor ECOG status to be associated with a higher risk of VTE. ${ }^{15-18,26}$

\section{Tumour Factors}

Tumour site and the presence of metastasis were the most commonly investigated tumor-related factors. Nine studies assessed tumor site. Five studies examined the risk of VTE in patients with gastric and pancreatic tumors compared with breast, colorectal, and head-neck tumors. Of these five studies, two prospective studies (reporting on the same patient cohort) found an increased risk of VTE in patients with gastric and pancreatic tumors compared with the other sites. $^{7,19}$ One multi-institutional prospective study compared only gastric tumors to other sites and found an increased risk of VTE in patients with gastric tumors. ${ }^{27}$ The evidence to suggest that lung, lymphoma, gynecologic, and genitourinary cancers grouped together were associated with a higher risk of VTE when compared with breast, colorectal and head-neck cancers in all three studies that made this comparison was inconclusive. ${ }^{7,20,22}$ Presence of metastasis was investigated in eleven studies. Of the seven patient cohorts represented across these studies, one retrospective study found that metastasis increased the risk of $\mathrm{VTE},{ }^{23}$ however, the remaining studies found no evidence of association.

\section{Biomarkers}

Eleven biomarkers were assessed to predict VTE. Prechemotherapy platelet count was explored in seven studies representing five patient cohorts and was found to be associated with a higher risk of VTE in four of these studies (or three patient cohorts). ${ }^{7,15,19,27}$ Prechemotherapy hemoglobin was explored in five studies representing four patient cohorts. One patient cohort found that low levels of hemoglobin or the use of erythropoietin stimulating agents increased the risk of VTE, ${ }^{7,19}$ while the other studies found no evidence of such association. Several individual studies identified novel biomarkers that were associated with VTE. The Ferroni et al. and Roselli et al. study group observed that high plateletlymphocyte ratio, impaired estimated glomerular filtration rate (eGFR), and decreased activated protein $C$ function were associated with an increased risk of VTE. ${ }^{14,17,18}$ Tafur et al. found low protein C levels to be a predictor of VTE, along with high factor VIII levels. ${ }^{24}$

\section{Treatment Factors}

Seventeen treatment-related factors for the development of VTE were identified. Gemcitabine and platinum-based compounds were the most commonly investigated treatment types. Across the seven studies (or four patient cohorts) exploring gemcitabine use, one study observed a higher 
Table 3 Classification of prognostic factors and number of studies reporting on each

\begin{tabular}{|c|}
\hline Patient factors \\
\hline $\begin{array}{l}\text { Age }(n=8) \text {, gender }(n=8) \text {, BMI }(n=4) \text {, ECOG }(n=8) \text {, Khorana score }(n=7) \text {, previous VTE }(n=1) \text {, vascular/lymphatic } \\
\text { macroscopic compression }(n=1) \text {, cardiovascular comorbidities }(n=1)\end{array}$ \\
\hline Tumour factors \\
\hline Tumour site $(n=9)$, metastasis $(n=11)$, time since cancer diagnosis $(n=1)$ \\
\hline Biomarkers \\
\hline $\begin{array}{l}\text { Prechemotherapy hemoglobin }(n=5) \text {, prechemotherapy white blood cell count }(n=4) \text {, prechemotherapy platelet count } \\
(n=7) \text {, low protein C activity }(n=1) \text {, high factor VIII activity }(n=1) \text {, ThromboPath change }(n=1) \text {, D-dimer levels }(n=4) \text {, fibrin } \\
\text { generation }(n=1) \text {, eGFR }(n=1) \text {, platelet/lymphocyte ratio }(n=1) \text {, neutrophil/lymphocyte ratio }(n=1)\end{array}$ \\
\hline Treatment Factors \\
\hline $\begin{array}{l}\text { Hospitalization }(n=1) \text {, adjuvant chemotherapy }(n=1) \text {, central venous catheter }(n=2) \text {, gemcitabine }(n=7) \text {, platinum }(n=7) \text {, } \\
\text { fluoropyrimidine }(n=5) \text {, irinotecan }(n=3) \text {, anthracycline }(n=5) \text {, docetaxel }(n=4) \text {, pemetrexed }(n=3) \text {, bevacizumab }(n=3) \text {, } \\
\text { herceptin }(n=3) \text {, anti-tyrosine kinase inhibitors }(n=6) \text {, endocrine/anti-hormonal therapy }(n=2) \text {, erythropoietin stimulating } \\
\text { agents }(n=4) \text {, prophylactic myeloid growth factors }(n=4) \text {, corticosteroids }(n=5)\end{array}$ \\
\hline Genetic factors \\
\hline $\begin{array}{l}\text { VEGFA-1190G/A A/A polymorphism }(n=1) \text {, VEGFA-1154G/A A/A polymorphism }(n=1) \text {, VEGFA-634G/C C/C polymorphism } \\
(n=1)\end{array}$ \\
\hline
\end{tabular}

risk of VTE in gemcitabine-treated patients compared with those who were administered other treatment regimens. ${ }^{20}$ Seven studies representing four patient cohorts investigated platinum-based compounds. All four patient cohorts reported the use of platinum-based compounds to increase the risk of VTE compared with non-platinum-based treatments. ${ }^{16,18,20,22,26}$ The most commonly investigated biological therapy was bevacizumab, which was reported in six studies representing two patient cohorts. One patient cohort found that bevacizumab was associated with a higher risk of VTE when compared with other treatments. ${ }^{16,17}$ Seven studies reporting on four patient cohorts investigated the effect of supportive drug use during chemotherapy, including erythropoietin stimulating agents, prophylactic myeloid growth factors and corticosteroids. The evidence across these studies to suggest that use of these drugs was associated with VTE development was inconclusive.

\section{Genetic Factors}

Ferroni et al. investigated genetic factors and observed that certain vascular endothelial growth factor (VEGFA) gene promoter variants, specifically, single nucleotide polymorphisms (SNPs) in these regions, were protective against VTE. ${ }^{16}$ The frequencies of each VEGFA SNP genotype in the study sample were comparable to a group of healthy controls.

\section{Discussion}

This systematic review identified pertinent prognostic factors in the literature for the development of VTE in patients with cancer undergoing systemic treatments. We identified the use of platinum-based chemotherapy compounds and poor performance status as important prognostic factors for VTE.

Currently, the most commonly recognized prognostic factors are those identified by Khorana et al. ${ }^{7} \mathrm{~A}$ recent systematic review and meta-analysis assessed the effectiveness of thromboprophylaxis in patients at an intermediate to high risk of VTE according to the Khorana score. For these patients, both direct oral anticoagulants (DOACs) and low molecular weight heparin (LMWH) significantly decreased the incidence of VTE. ${ }^{28}$ However, the administration of DOACs doubled the risk of major bleeding compared with the placebo group. Additionally, several trials in the review reported a higher risk of clinically relevant non-major bleeding in groups receiving DOAC or LMWH compared with placebo. ${ }^{28}$ Thus, it is important to carefully consider the risks associated with anticoagulants against the benefits, and to appropriately identify patients who are at risk of developing VTE. One of the limitations of the Khorana score was that most patients used to derive the scoring model had a good performance status. $^{29}$ However, in more recent studies identified by this review, poor performance status (i.e., ECOG performance status $\geq 2$ ) increased the risk of VTE and should be considered independently when assessing patients for VTE risk.

Given these limitations, modified versions of the Khorana score which take into consideration additional clinical and biomarker-based variables have been created, such as the Vienna CATS $^{9}$ and PROTECHT ${ }^{30}$ scores. One multinational, prospective cohort study observed greater predictive power by these two scores compared with the Khorana score, mainly due to the addition of D-dimer levels and chemotherapy type as variables. ${ }^{20}$ However, drawbacks of these scores include difficulty measuring D-dimer in clinical practice and an increased complexity of administering scores as more items are added. In the same study, the incidence of VTE was still appreciable in patients classified as low-risk by these scores, with 6-month rates of 5-6\% in low-risk patients and $8-10 \%$ in high-risk patients. ${ }^{20}$

Metastasis has previously been reported as a significant prognostic factor for VTE. ${ }^{1,4}$ In the current review, however, 
the evidence to suggest that metastasis was associated with a higher risk of VTE was inconclusive. Chew et al. conducted one of the largest database studies of cancer-associated thrombosis to date and found that for all cancer types analyzed, metastatic disease increased the risk of VTE compared with localized disease. ${ }^{4}$ However, the risk of VTE is exacerbated by the administration of certain anticancer treatments. ${ }^{18,30-32}$ Thus, the effect of metastasis may have been lost when adjusted for treatment type in the included studies. Another possible explanation is the exclusion of patients with leukemia. Previous studies evaluating metastasis on the risk of VTE included patients with non-solid tumors. ${ }^{1,31}$ Given the high incidence of VTE in patients with leukemia, ${ }^{4,33}$ inclusion of these patients may have acted as a confounding factor. Additionally, metastasis did not have a consistent definition across individual studies, which may explain the lack of association with VTE. Some studies included locally advanced disease in their reference group (i.e., non-metastatic group), while some studies did not specify. Given that both locally advanced and distant cancer stages are independent predictors of VTE, ${ }^{34}$ this may partly describe the low difference in VTE risk between metastatic and non-metastatic groups in the included studies.

Gemcitabine and platinum-containing chemotherapy were the most commonly reported treatment-related prognostic factors for VTE. Similar to previous studies, ${ }^{35-37}$ we found that the use of platinum-based compounds was associated with an increased risk of VTE across the included studies. The association between the use of gemcitabine and VTE was less conclusive, despite previous studies suggesting that this treatment increased the risk of VTE, ${ }^{37,38}$ which may be explained by adjustment factors and potential confounding.

There are several important limitations to consider. First, all of the included studies were assessed as having a moderate to high risk of bias. One of the main sources of bias was potential confounding. Although studies investigated the risk of chemotherapy types and supportive drugs, details such as dose and duration of these treatments were unavailable, which may have acted as sources of confounding. Other confounding variables may have been missed, as most studies did not provide an adequate explanation of how their multivariable models were built and how they selected which factors to include in their model. Additionally, there was poor reporting on the analysis models which acted as another major source of bias. Many studies did not describe how they defined certain prognostic factors in their multivariable analyses, nor how these statistical models were developed. Second, while some variables were prognostic in individual studies, we were not able to confirm their association with VTE in this review. This was due to large differences in the analysis of prognostic factors across studies, as our findings were based on the summary results of individual studies. Although we controlled for at least two types of adjustment factors (i.e., tumor type and/or metastasis), studies used a diverse set and number of adjustment factors in their analyses to estimate the effect of each prognostic factor. Moreover, prognostic factors had varied definitions and cut-offs across studies or lacked a clear explanation of how they were defined in the multivariable analysis altogether. Different summary measures were also used across studies. Due to these limitations, we were unable to perform meta-analysis for the prognostic factors that were identified. In this review, only adjusted risk estimates, with adjustment factors defined a priori, were collected. Although this partly standardized the risk estimates that were collected, a large number of studies were excluded on the basis of this criteria. Thus, risk factors that may be prognostic in excluded individual studies are not reported. Finally, many studies relied on a low number of VTE events or very few participants who possessed a prognostic factor, which may have overestimated the effect of some factors in the individual studies.

Overall, many prognostic factors were identified in this review, but the effect of these factors on VTE risk is inconclusive. These findings should be interpreted in light of the high risk of bias of included studies and heterogeneity across studies. There was an overall poor quality in reporting which is an area for improvement for future prognostic studies.

\section{Author Contributions}

Study concept and design: S Parpia, P Serrano, S Lee; Data acquisition: S Lee, A Shenoy; Data analysis and interpretation: All authors; Drafting and revising of the manuscript: All authors; Final approval of the manuscript version for publication: All authors.

\section{Funding}

This research did not receive any specific grant from funding agencies in the public, commercial, or not-forprofit sectors.

\section{Conflicts of Interest}

None declared.

\section{Acknowledgments}

None.

\section{References}

1 Blom JW, Vanderschoot JP, Oostindiër MJ, Osanto S, van der Meer FJ, Rosendaal FR. Incidence of venous thrombosis in a large cohort of 66,329 cancer patients: results of a record linkage study. J Thromb Haemost 2006;4(03):529-535. Doi: 10.1111/j.15387836.2006.01804.x

2 Heit JA, Silverstein MD, Mohr DN, Petterson TM, O'Fallon WM, Melton LJ III. Risk factors for deep vein thrombosis and pulmonary embolism: a population-based case-control study. Arch Intern Med 2000;160(06):809-815. Doi: 10.1001/archinte.160.6.809

3 Sallah S, Wan JY, Nguyen NP. Venous thrombosis in patients with solid tumors: determination of frequency and characteristics. Thromb Haemost 2002;87(04):575-579

4 Chew HK, Wun T, Harvey D, Zhou H, White RH. Incidence of venous thromboembolism and its effect on survival among patients with common cancers. Arch Intern Med 2006;166(04): 458-464. Doi: 10.1001/archinte.166.4.458

5 Khorana AA, Francis CW, Culakova E, Kuderer NM, Lyman GH. Thromboembolism is a leading cause of death in cancer patients receiving outpatient chemotherapy. J Thromb Haemost 2007;5 (03):632-634. Doi: 10.1111/j.1538-7836.2007.02374.x 
6 Lyman GH, Eckert L, Wang Y, Wang H, Cohen A. Venous thromboembolism risk in patients with cancer receiving chemotherapy: a real-world analysis. Oncologist 2013;18(12):1321-1329. Doi: 10.1634/theoncologist.2013-0226

7 Khorana AA, Kuderer NM, Culakova E, Lyman GH, Francis CW. Development and validation of a predictive model for chemotherapy-associated thrombosis. Blood 2008;111(10):4902-4907. Doi: 10.1182/blood-2007-10-116327

8 Mulder FI, Candeloro M, Kamphuisen PW, et al; CAT-prediction collaborators. The Khorana score for prediction of venous thromboembolism in cancer patients: a systematic review and metaanalysis. Haematologica 2019;104(06):1277-1287. Doi: 10.3324/ haematol.2018.209114

9 Ay C, Dunkler D, Marosi C, et al. Prediction of venous thromboembolism in cancer patients. Blood 2010;116(24):5377-5382. Doi: 10.1182/blood-2010-02-270116

10 Barni S, Labianca R, Agnelli G, et al. Chemotherapy-associated thromboembolic risk in cancer outpatients and effect of nadroparin thromboprophylaxis: results of a retrospective analysis of the PROTECHT study. J Transl Med 2011;9:179. Doi: 10.1186/1479-5876-9-179

11 Moher D, Liberati A, Tetzlaff J, et al. Preferred reporting items for systematic reviews and meta-analyses: the PRISMA statement. Ann Intern Med 2009;151(04):264-9, W64. Doi: 10.7326/00034819-151-4-200908180-00135

12 Wun T, White RH. Epidemiology of cancer-related venous thromboembolism. Best Pract Res Clin Haematol 2009;22(01):9-23. Doi: $10.1016 /$ j.beha.2008.12.001

13 Hayden JA, van der Windt DA, Cartwright JL, Côté P, Bombardier C. Assessing bias in studies of prognostic factors. Ann Intern Med 2013;158(04):280-286. Doi: 10.7326/0003-4819-158-4201302190-00009

14 Ferroni P, Guadagni F, Laudisi A, et al. Estimated glomerular filtration rate is an easy predictor of venous thromboembolism in cancer patients undergoing platinum-based chemotherapy. Oncologist 2014;19(05):562-567. Doi: 10.1634/theoncologist.2013-0339

15 Ferroni P, Guadagni F, Riondino S, et al. Evaluation of mean platelet volume as a predictive marker for cancer-associated venous thromboembolism during chemotherapy. Haematologica 2014; 99(10):1638-1644. Doi: 10.3324/haematol.2014.109470

16 Ferroni P, Palmirotta R, Riondino S, et al. VEGF gene promoter polymorphisms and risk of VTE in chemotherapy-treated cancer patients. Thromb Haemost 2016;115(01):143-151. Doi: 10.1160/ TH15-03-0259

17 Ferroni P, Riondino S, Formica V, et al. Venous thromboembolism risk prediction in ambulatory cancer patients: clinical significance of neutrophil/lymphocyte ratio and platelet/lymphocyte ratio. Int J Cancer 2015;136(05):1234-1240. Doi: 10.1002/ ijc.29076

18 Roselli M, Ferroni P, Riondino S, et al. Impact of chemotherapy on activated protein C-dependent thrombin generation-association with VTE occurrence. Int J Cancer 2013;133(05):1253-1258. Doi: 10.1002/ijc. 28104

19 Khorana AA, Francis CW, Culakova E, Lyman GH. Risk factors for chemotherapy-associated venous thromboembolism in a prospective observational study. Cancer 2005;104(12):2822-2829. Doi: 10.1002/cncr.21496

20 van Es N, Di Nisio M, Cesarman G, et al. Comparison of risk prediction scores for venous thromboembolism in cancer patients: a prospective cohort study. Haematologica 2017;102 (09):1494-1501. Doi: 10.3324/haematol.2017.169060

21 van Es N, Hisada Y, Di Nisio M, et al. Extracellular vesicles exposing tissue factor for the prediction of venous thromboembolism in patients with cancer: A prospective cohort study. Thromb Res 2018;166:54-59. Doi: 10.1016/j.thromres.2018.04.009
22 Di Nisio M, van Es N, Rotunno L, et al. Long-term performance of risk scores for venous thromboembolism in ambulatory cancer patients. J Thromb Thrombolysis 2019;48(01):125-133. Doi: 10.1007/s11239-019-01845-6

23 Abdel-Razeq H, Mansour A, Abdulelah H, et al. Thromboembolic events in cancer patients on active treatment with cisplatinbased chemotherapy: another look!. Thromb J 2018;16:2. Doi: 10.1186/s12959-018-0161-9

24 Tafur AJ, Dale G, Cherry M, et al. Prospective evaluation of protein $\mathrm{C}$ and factor VIII in prediction of cancer-associated thrombosis. Thromb Res 2015;136(06):1120-1125. Doi: 10.1016/j. thromres.2015.10.004

25 Arpaia G, Carpenedo M, Verga M, et al. D-dimer before chemotherapy might predict venous thromboembolism. Blood Coagul Fibrinolysis 2009;20(03):170-175. Doi: 10.1097/MBC.0b013e32831bc2de

26 Vergati M, Della-Morte D, Ferroni P, et al. Increased risk of chemotherapy-associated venous thromboembolism in elderly patients with cancer. Rejuvenation Res 2013;16(03):224-231. Doi: 10.1089/rej.2013.1409

27 Gerotziafas GT, Taher A, Abdel-Razeq H, et al; COMPASS-CAT Working Group. A Predictive Score for Thrombosis Associated with Breast, Colorectal, Lung, or Ovarian Cancer: The Prospective COMPASS-Cancer-Associated Thrombosis Study. Oncologist 2017;22(10):1222-1231. Doi: 10.1634/theoncologist.2016-0414

28 Bosch FTM, Mulder FI, Kamphuisen PW, et al. Primary thromboprophylaxis in ambulatory cancer patients with a high Khorana score: a systematic review and meta-analysis. Blood Adv 2020;4 (20):5215-5225. Doi: 10.1182/bloodadvances.2020003115

29 Doggen CJ. Thromboprophylaxis in cancer outpatients. Blood 2008;111(10):4833. Doi: 10.1182/blood-2008-02-137760

30 Verso M, Agnelli G, Barni S, Gasparini G, LaBianca R. A modified Khorana risk assessment score for venous thromboembolism in cancer patients receiving chemotherapy: the Protecht score. Intern Emerg Med 2012;7(03):291-292. Doi: 10.1007/s11739012-0784-y

31 Blom JW, Doggen CJ, Osanto S, Rosendaal FR. Malignancies, prothrombotic mutations, and the risk of venous thrombosis. JAMA 2005;293(06):715-722. Doi: 10.1001/jama.293.6.715

32 Haddad TC, Greeno EW. Chemotherapy-induced thrombosis. Thromb Res 2006;118(05):555-568. Doi: 10.1016/j. thromres.2005.10.015

33 Elliott MA, Wolf RC, Hook CC, et al. Thromboembolism in adults with acute lymphoblastic leukemia during induction with Lasparaginase-containing multi-agent regimens: incidence, risk factors, and possible role of antithrombin. Leuk Lymphoma 2004; 45(08):1545-1549. Doi: 10.1080/10428190410001693588

34 Dickmann B, Ahlbrecht J, Ay C, et al. Regional lymph node metastases are a strong risk factor for venous thromboembolism: results from the Vienna Cancer and Thrombosis Study. Haematologica 2013;98(08):1309-1314. Doi: 10.3324/haematol.2012.073338

35 Alahmari AK, Almalki ZS, Alahmari AK, Guo JJ. Thromboembolic Events Associated with Bevacizumab plus Chemotherapy for Patients with Colorectal Cancer: A Meta-Analysis of Randomized Controlled Trials. Am Health Drug Benefits 2016;9(04):221-232

36 Li LJ, Chen DF, Wu GF, et al. Incidence and risk of thromboembolism associated with bevacizumab in patients with non-small cell lung carcinoma. J Thorac Dis 2018;10(08):5010-5022. Doi: $10.21037 /$ jtd.2018.07.09

37 Numico G, Garrone O, Dongiovanni V, et al. Prospective evaluation of major vascular events in patients with nonsmall cell lung carcinoma treated with cisplatin and gemcitabine. Cancer 2005; 103(05):994-999. Doi: 10.1002/cncr.20893

38 Dasanu CA. Gemcitabine: vascular toxicity and prothrombotic potential. Expert Opin Drug Saf 2008;7(06):703-716. Doi: $10.1517 / 14740330802374262$ 